http://jmscr.igmpublication.org/home/ ISSN (e)-2347-176x ISSN (p) 2455-0450 crossref DOI: https://dx.doi.org/10.18535/jmscr/v9i9.05

\author{
Journal Of Medical Science And Clinical Research

\title{
A Case of Systemic Lupus Erythematosus Presenting as Lupus Nephritis
}

\author{
Authors \\ Dr Kamini Randhawa ${ }^{1}$, Dr Lucky Kumar ${ }^{2}$, Dr Meenakshi Rana ${ }^{3 *}$ \\ ${ }^{1}$ Junior Resident, Department of Anaesthesiology, IGMC Shimla \\ ${ }^{2}$ Junior Resident, Department of Internal Medicine, IGMC Shimla \\ ${ }^{3}$ Junior Resident, Department of Obs and Gynaecology, IGMC Shimla \\ *Corresponding Author \\ Dr Meenakshi Rana \\ Junior Resident, Department of Obs and Gynaecology, IGMC Shimla, India
}

\begin{abstract}
Systemic Lupus Erythematosus (SLE), is a multisystemic disease with an unknown etiology. It is especially frequent in females. SLE has a vasculitic feature and may affect many systems of the body ${ }^{1}$. SLE is a chronic inflammatory disease that affects the kidneys in about 50\%of patients. Lupus nephritis is a major risk factor for overall morbidity and mortality in $S L E^{2}$. We present a case of 35 year old female admitted with chief complaint of fever, swelling of both feet and decreased urine output.
\end{abstract}

\section{Introduction}

Systemic lupus erythematosus (SLE), is an inflammatory connective tissue disorder that occurs Commonly in females ${ }^{1}$. The four main types of lupus are neonatal and pediatric lupus erythematosus (NLE); discoid lupus erythematosus (DLE); drug induced lupus (DIL); and systemic lupus erythematosus (SLE). ${ }^{3}$ The pathogenesis of SLE consists of antibodies (antinuclear antibody, antids DNA, anti-histone antibody) and immune complexes, resulting in damage to many tissues and organs. ${ }^{1}$ General signs and symptoms observed in SLE include fever, fatigue, and weight loss. The skin, musculoskeletal system, and pulmonary system are primarily affected. ${ }^{3}$ American College of Rheumathology's (ACR) criteria are used to draw thediagnose for SLE. At least 4 criteria of 11 must be present for diagnosis ${ }^{1}$.

\section{Case Report}

A 35 year old female admitted with chief complaint of fever for 5days 25 days back, Swelling of both feet for 21 days and decrease urine output for 7 days. Feverish feeling associated with chills and rigors, intermittent, relieved by medications, Undocumented, daily. No history of nasal discharge, ear discharge, headache, cough, nausea, vomiting, loose motions, rashes, joint swelling, abdominal distention, burning micturition. Swelling both feet for 21 days which was Insidious in onset, gradually progressive associated with periorbital puffiness at morning time and not associated with shortness of breath or discomfort on lying down. Decreased urine output for 7 days which was Insidsious in onset, progressive in nature. Not associated with cola / froathy / cloudy urine but associated with decreased appetite, nausea. Her 
past history, medical history and family history was not significant. On general examination patient was conscious, cooperative, oriented to time, place, person, Malar rash present .Patient was short stature (familial), pallor and pedal edema was present and J V P was raised. There was no Icterus, cyanosis and lymphaednopathy. Her vitals were pulse of $86 / \mathrm{min}$, B P $140 / 80$ mmhg, RR 16 / min, SPO2 $94 \%$ and Temprature. $98.4 * \mathrm{~F}$. Examination of other system was not significant. Provisional diagnosis of acute nephritic/nephrotic syndrome and etiology of SLE and PSGN were made. Hematological investigation show $\mathrm{Hb} 9.8 \mathrm{gm} / \mathrm{dl}$,TLC 10000/microl, Neutrophils $80 \%$, lymphocytes 15 $\%$, Platelet : 4,80,000, ESR $80 \mathrm{~mm} 1 \mathrm{stHr}, \mathrm{P} / \mathrm{S}$ show normocytic, normochromic and RBS of $78 \mathrm{mg} / \mathrm{dl}$. RFT show Urea $60 \mathrm{mg} / \mathrm{dl}$ and Creat 4.1 $\mathrm{mg} / \mathrm{dl}$. ABG show metabolic acidosis with respiratory compensation. Urine analysis show WBCs: 8-10 PHF, RBCs: 8-10 PHF, Protein : 3+ and Glucose was absent. $24 \mathrm{hrs}$ Protein was 2.5gm.CXR and ECG was normal .USG (KUB) revealed RT Kidney $9.58 * 4 \mathrm{CM}$, LT kidney 10* 4.5 CM and B/l MRD. Immunological investigation show ANA Positive $1: 640$,DS DNA Positive, C3 level was 36 (normal 81 -120), DCT Positive and ASO was Negative. Final diagnosis of SLE presented as lupus nephritis were made. Treatment was given with IV methylprednisolone $0.25 \mathrm{~g} /$ day for first 3 days followed by oral prednisone $1 \mathrm{mg} / \mathrm{kg} / \mathrm{day}$. The patient's complaints relieved with prednisolon treatment.

\section{Discussion}

SLE is a multisystemic disease which effect many organs. Auto antibodies are responsible for the multisystemic involvement. ${ }^{1} \mathrm{LN}$ is a major risk factor for morbidity and mortality in SLE and $10 \%$ of patients with LN will develop ESRD. The clinical manifestations of LN are often subtle and most commonly will be discovered by examination of the urine as opposed to physical examination. All patients with SLE should be evaluated for kidney involvement at initial diagnosis and at least yearly thereafter even if they do not have symptoms of kidney disease.

Evaluation include a urinalysis and measurement of kidney function, generally a serum creatinine concentration or $\mathrm{eGFR}^{2}$. Lupus nephritis is an important prognostic indicator and it can lead to nephrotic syndrome and renal failure in developed patients by autoantibodies. Lupus nephritis develops in $50 \%$ of systemic lupus patients. Nephritis is divided into 5 pathologic classication groups by World Health Organisation (Table1) One of the laboratory findings of lupus nephritis is proteinuria. . Treatment include IV methylprednisolone $0.25 \mathrm{~g} /$ day for first 3 days followed by oral prednisone $1 \mathrm{mg} / \mathrm{kg} / \mathrm{day}$.

\section{References}

1. Klippel JH, Dieppe PA, et al: Rheumatology, second edition, 1998, volume two, section 7, page 2,1-2,8

2. Salem Almaani, Alexa Meara, and Brad H. Rovin. Update on lupus nephritis . Clin J Am Soc Nephrol; 2016:1-11

3. William Maidhof et al .Lupus : An overview of disease and management option.P T :2012;37(4) 